\title{
Nanoscale Phenomena
}

National Cancer Institute

\section{Source}

National Cancer Institute. Nanoscale Phenomena. NCI Thesaurus. Code C62347.

Events that occur specifically at the nanoscale level due to changes in physical properties and interactions. At this level, phenomena are controlled primarily by Coulombic forces, quantum mechanics, and the random thermal motion of particles. 